\title{
Estimating the flexural rigidity of Arabidopsis inflorescence stems: Free-vibration test vs. three-point bending test
}

\author{
Miyuki T. Nakata', Mao Nakao', Asuka Denda², Yusuke Onoda³, Haruko Ueda², \\ Taku Demura, ${ }^{1, *}$ \\ ${ }^{1}$ Division of Biological Science, Graduate School of Science and Technology, Nara Institute of Science and Technology \\ (NAIST), Ikoma, Nara 630-0192, Japan; ${ }^{2}$ Department of Biology, Faculty of Science and Engineering, Konan University, Kobe, \\ Hyogo 658-8501, Japan; ${ }^{3}$ Graduate School of Agriculture, Kyoto University, Kyoto, Kyoto 606-8502, Japan \\ *E-mail: demura@bs.naist.jp Tel: +81-743-72-5460 Fax:+81-743-72-5469
}

Received August 31, 2020; accepted December 14, 2020 (Edited by S. Sawa)

\begin{abstract}
The mechanical strength of a plant stem (a load-bearing organ) helps the plant resist drooping, buckling and fracturing. We previously proposed a method for quickly evaluating the stiffness of an inflorescence stem in the model plant Arabidopsis thaliana based on measuring its natural frequency in a free-vibration test. However, the relationship between the stiffness and flexural rigidity of inflorescence stems was unclear. Here, we compared our previously described free-vibration test with the three-point bending test, the most popular method for calculating the flexural rigidity of $A$. thaliana stems, and examined the extent to which the results were correlated. Finally, to expand the application range, we present an example of a modified free-vibration test. Our results provide a reference for improving estimates of the flexural rigidity of $A$. thaliana inflorescence stems.
\end{abstract}

Key words: Arabidopsis thaliana, flexural rigidity, free-vibration test, inflorescence stem, three-point bending test.

The aerial parts of a land plant are constantly exposed to mechanical stress from the external environment, e.g., gravity, wind, animals, and surrounding plants. A stem is a load-bearing organ whose mechanical strength provides the plant with resistance to drooping, buckling and fracture. The extent of resistance against deformation by the bending force of stems is determined by flexural rigidity $(E I)$. Vibration characteristics are determined by stiffness $(k)$ of bending (de Langre 2019). These values are related, as greater stiffness causes a higher natural frequency of vibration. Stem vibration is caused by the bending force, and thus the stiffness value $k$ is associated with EI. Several methods are used to estimate the EI values of plant organs, including the three-point bending test and the free-vibration method (Niklas and Spatz 2012; Shah et al. 2017; Spatz and Speck 2002; Zebrowski 1991). The bending test allows $E I$ to be calculated directly but requires an expensive machine equipped with a load cell and a linear actuator (for example, a material testing machine and a creep meter). By contrast, a vibration test can be performed using relatively inexpensive tools, but $E I$ is estimated indirectly based on the natural frequency of vibration.

Arabidopsis thaliana is the most widely studied model land plant. Although A. thaliana is an annual herbaceous plant that forms rosette leaves, it is used as a model for woody plants due to its advantages for investigating molecular mechanisms (e.g., its short life cycle, availability of genetic information and easy genetic manipulation). The inflorescence stem of $A$. thaliana is a load-bearing organ, making it suitable for studying the mechanical aspects of plants (Strabala and Macmillan 2013). The mechanical properties of wild-type, mutant and transgenic A. thaliana plants have been analyzed using various machine-mediated tests (reviewed by Brulé et al. 2016), including the three-point bending test to calculate $E I$ and the bending modulus in cell wall-related mutants (Bichet et al. 2001; Jones et al. 2001; MacMillan et al. 2010, 2013; Paul-Victor and Rowe 2011; Turner and Somerville 1997; Zhu et al. 2015). A more accessible method for quickly evaluating $k$ of an inflorescence stem was recently proposed based on natural frequency, as determined from a free-vibration test (Nakata et al. 2018). However, the relationship between the $k$ and $E I$ values of $A$. thaliana inflorescence stems remains to be solved.

Here, we compare the free-vibration test and threepoint bending test. We generated a regression equation for calculating EI based on $k$ determined by a freevibration test (experiment $\mathrm{A}$ : exA) and modified the free-vibration test method for use in stems with various phenotypes (experiment B: exB). 
We performed regression analysis of the relationship between $E I$ and $k$ using 53 natural accessions of $A$. thaliana from the 1001 Genomes Project (CS78942; Supplementary Table S1; The 1001 Genomes Consortium 2016) established to have wide mechanical variability (MacMillan et al. 2013). We used A. thaliana accession Columbia-0 (Col-0) for the modified free-vibration test. The seeds were surface sterilized, sown on solid MS medium (1x Murashige and Skoog salts, 1\% sucrose, $\mathrm{pH}$ 5.7) containing $0.05 \% \mathrm{MES}-\mathrm{KOH}$, vitamin mix and $0.25 \%$ gellan gum (for exA) or $0.5 \mathrm{M}$ MES-KOH, $0.01 \%$ myo-inositol and $0.5 \%$ gellan gum (for exB). The plates were stored at $4^{\circ} \mathrm{C}$ in the dark for 3 days, and the plants were grown at $22^{\circ} \mathrm{C}$ under long-day conditions (16-h light/8-h dark) (for exA) or under continuous light (for exB). Two-week-old plants were transplanted to culture soil with vermiculite (for exA) or vermiculite alone (for exB) and grown under long-day conditions at $22^{\circ} \mathrm{C}$ until their height exceeded $20 \mathrm{~cm}$ (for exA) or reached $15-20 \mathrm{~cm}$ (for exB). The primary stems were harvested and immediately used for the free-vibration test. For exA, after performing the original version of the free-vibration test, the stems were soaked in water and their surfaces wiped briefly with a paper towel before performing the three-point bending test.

For exA, the free-vibration test was performed as previously described (Nakata et al. 2018) using $10-\mathrm{cm}$ fragments from the base of each inflorescence stem. The camera of an iPhone $6 \mathrm{~s}$ plus was used to capture 240-fps (frame per second) videos with the high speed-shooting function (which equals the slomo mode) of Photo App. The captured videos were analyzed using the Python program AraVib, and damped natural frequency $f_{d}$ was automatically calculated. We previously reported that there is only a very small difference between natural frequency and damped natural frequency in $A$. thaliana stems (Nakata et al. 2018). In this study, we treated damped natural frequency $f_{d}$ as equivalent to natural frequency $f_{n}$ for convenience. Stiffness $k$ was calculated using the following equation:

$$
k=\left(2 \pi f_{d}\right)^{2} m_{e q}
$$

where $m_{e q}$ represents the equivalent mass of the oscillator. $m_{e q}$ was calculated using the following equation:

$$
m_{e q}=0.245 \times 0.9 \times[\text { Fresh weight }]
$$

where [Fresh weight] represents the fresh weight of the $10-\mathrm{cm}$ fragment weighed with a precision balance before capturing the video. The weight of the $10-\mathrm{cm}$ fragment was the order of $\mathrm{mg}$, and when $k$ was calculated, the unit of $m_{e q}$ was converted from $\mathrm{mg}$ to $\mathrm{kg}$.

For the three-point bending test, a Rheoner II Creep Meter (RE2-33005C) equipped with $20 \mathrm{~N}$ load cell (Yamaden, Tokyo, Japan) was used. For the three-point
A
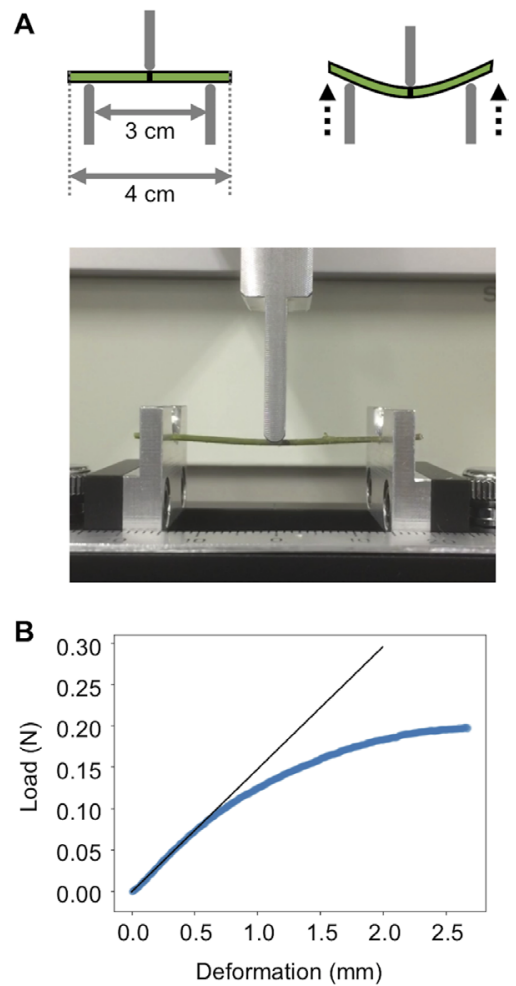

Figure 1. Overview of the three-point bending test. (A) Schematic diagrams (the upper panels) and an image (the lower panel) of the three-point bending test. (B) Example of a load-deformation curve generated using the three-point bending test.

bending test, the optional jig FX-3305B-3L was attached to the creep meter. The sensitivity of the load cell was set to $10 \mathrm{x}$ amplification. The $4-\mathrm{cm}$ fragment from the base was attached orthogonally to a plunger on the upper side and two supports $(3 \mathrm{~cm}$ apart from each other) on the lower side (Figure 1A). The lower supports were moved upwards at a constant speed $\left(0.05 \mathrm{~mm} \mathrm{~s}^{-1}\right)$. During this time, the load was sequentially measured by the plunger connected to the load cell (Figure 1A). The values were used to generate a load-deformation curve. Since we commonly observed a linear correlation between the load and the deformation value during $0.0-0.6 \mathrm{~mm}$ of deformation of the tested stems, we used a Python program to automatically calculate the coefficient based on the linear regression model (Figure 1B). Based on this coefficient, we estimated $E I$ using the following equation (Niklas and Spatz 2012; Onoda et al. 2015):

$$
E I=\frac{L^{3}}{48} \frac{F}{\delta}
$$

where $F$ represents the load, $\delta$ represents the displacement, and $L$ represents the span $(3 \mathrm{~cm}$ in this study). $F / \delta$ corresponds to the initial slope from the loaddeformation curve.

To generate a regression equation between $k$ and $E I$, we examined 137 plants from the 1001 Genomes collection using the free-vibration test and the three- 
point bending test. As expected, the $k$ and $E I$ values showed wide variations (Figure 2A). The $k$ ranged from $0.027-0.810\left(\mathrm{~N} \mathrm{~m}^{-1}\right)$ and the EI ranged from 5.18$148.16\left(\mathrm{~N} \mathrm{~mm}^{2}\right)$, indicating that both parameters varied approximately 30 -fold across the plants. Importantly, we detected a strong positive correlation between $k$ and EI (Figure $2 \mathrm{~A} ; r^{2}=0.8582$ ). The regression equation was estimated as $E I=154.8 k+6.2$ by the least square method. To evaluate the precision of the estimation, we calculated the relative error between EI derived from the threepoint bending test and EI predicted based on $k$. The relative error was calculated using the following equation:

$$
[\text { Relative error }]=\frac{[\text { Measured } E I]-[\text { Predicted } E I]}{[\text { Predicted } E I]}
$$

The distribution of relative error is shown in Figure 2B. In this experiment, $50 \%$ of the samples fell within an error range of approximately $15 \%$, and $75 \%$ fell within an error range of approximately $25 \%$, indicating that the error range was much smaller than the range of individual differences.

Our data thus revealed a strong correlation between $k$ and $E I$. Using regression analysis, we generated an equation to estimate $E I$ from $k$. The $E I$ values calculated from the three-point bending test differed from those predicted from $k$. There are several possible reasons for this. Estimations of both $E I$ and $k$ could have artificial errors. In the vibration test, the prerequisite for estimating $k$ is that the tested stems are straight and have a uniform distribution of mass. According to Nakata et al. (2018), the basal $10-\mathrm{cm}$ fragments of $20-30-\mathrm{cm}$ tall Col-0 plants tend to be linear in shape and have little bias in terms of mass. However, whether this is true for the other accessions examined in the current study has not been investigated. Indeed, natural variations in mechanical and cell wall properties were previously observed in a study of 12 A. thaliana accessions (MacMillan et al. 2013). In addition, in the threepoint bending test, the ovalization of an inflorescence stem in the transverse plane might negatively affect EI estimations (Niklas and Spatz 2012). Finally, the properties of the samples used in the two tests might have differed: because we needed to use the same samples in both tests, we used freshly harvested stems in the free-vibration test and water-soaked samples in the three-point bending test. Freshly harvested samples are usually used for bending tests of $A$. thaliana stems to reduce the effects of water loss (Paul-Victor and Rowe 2011; Turner and Somerville 1997). Nevertheless, the errors of the predicted $E I$ values were much smaller than the individual differences in these values. Therefore, the regression equation generated in this study provided a rough prediction of the $E I$ values obtained thus far.

Of the artificial errors described above, we focused on reducing the negative effects of variations in shape
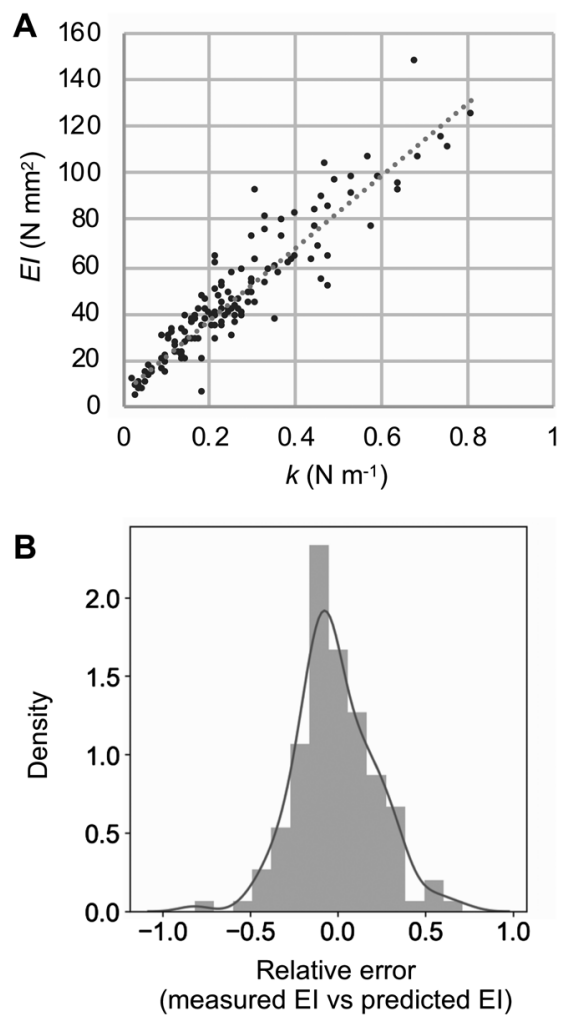

Figure 2. Correlation between stiffness $k$ and flexural rigidity EI. (A) Results of regression analysis of stiffness $k$ and flexural rigidity $E I$. (B) Density plot of the relative differences between predicted $E I$ and measured EI values.

and mass distribution, as well as the use of our method to examine various traits of $A$. thaliana stems. We developed a modified free-vibration test using shorter stem fragments (which equals exB). The following modifications were made to the free-vibration test: we used $6-\mathrm{cm}$ fragments, we marked the $1-\mathrm{cm}$ position from the apical end with red dye, we sandwiched the $1-\mathrm{cm}$ region from the base between two metal blocks (Supplementary Figure S1A) and we pulled the apical end of the stem sideways with a bamboo skewer (Supplementary Figure S1B) and released it after filming began (Figure 3A, Supplementary Figure S1C). Using the modified method, it appeared that the vibration speed increased (Figure 3A, B) and the oscillation was quickly damped (Figure 3B). Based on these observations, we altered the size of the Hanning window from 240 frames (1s) to 80 frames $(1 / 3 \mathrm{~s})$ in the AraVib program. In the original version, the damped natural frequency was $<30$ $\mathrm{Hz}$ (Nakata et al. 2018), whereas in the modified version, the damped natural frequency was $30-50 \mathrm{~Hz}$ (Figure $3 \mathrm{C})$. The ratio of the damped natural frequency to the sampling frequency $(30-50: 240)$ was reasonable for frequency analysis according to the Nyquist-Shannon sampling theorem (Nyquist 1928; Shannon 1949). These data indicate that the natural frequency of shorter stems could be analyzed using the modified version of the free- 
A



B
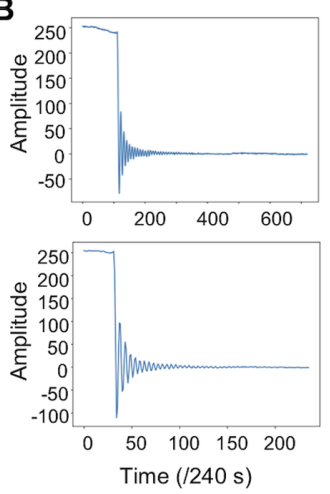

C
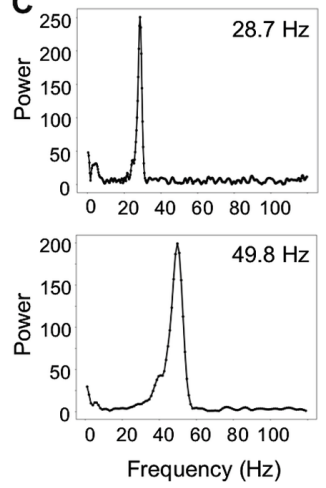

Figure 3. The modified free-vibration test shows the higher-speed and quickly-damped oscillation. (A) Frames of a high-speed video obtained during the free vibration of stems. " $t$ " represents time after the start of vibration. (B) Examples of vibration waveforms. (C) Examples of FFT power spectra. In (A-C), the upper panels show the results of the original test using 10-cm fragments and the lower panels show the results of the modified test using 6-cm fragments.

vibration test. We plan to compare $k$ from this modified method with that from the original version for the usage to reduce the negative effects of variations in shape and mass distribution and expand its application range.

\section{Acknowledgements}

We thank the Arabidopsis Biological Resource Center for providing the seeds of natural accessions from the 1001 Genomes Project, Eriko Tanaka (NAIST, Japan) for technical support and Ko Kato (NAIST, Japan), Tadashi Kunieda (NAIST, Japan) and Satoru Tsugawa (NAIST, Japan) for technical advice.

This work was supported by MEXT KAKENHI Grant-inAid for Scientific Research on Innovative Areas "Plant-Structure Optimization Strategy” Grant Numbers JP19H05365 (to Y.O.), JP18H05496 (to H.U.), JP18H05484 and JP18H05489 (to T.D.) and by JSPS KAKENHI Grant Numbers JP19K23753 (to M.N.) and JP19K06732 (to H.U.).

\section{References}

Bichet A, Desnos T, Turner S, Grandjean O, Höfte H (2001) BOTERO1 is required for normal orientation of cortical microtubules and anisotropic cell expansion in Arabidopsis. Plant J 25: 137-148

Brulé V, Rafsanjani A, Pasini D, Western TL (2016) Hierarchies of plant stiffness. Plant Sci 250: 79-96

de Langre E (2019) Plant vibrations at all scales: A review. J Exp Bot 70: 3521-3531

Jones L, Ennos AR, Turner SR (2001) Cloning and characterization of irregular xylem4 (irx4): A severely lignin-deficient mutant of Arabidopsis. Plant J 26: 205-216

MacMillan CP, Mansfield SD, Stachurski ZH, Evans R, Southerton SG (2010) Fasciclin-like arabinogalactan proteins: Specialization for stem biomechanics and cell wall architecture in Arabidopsis and Eucalyptus. Plant J 62: 689-703

MacMillan CP, O’Donnell PJ, Smit AM, Evans R, Stachurski ZH, Torr K, West M, Baltunis J, Strabala TJ (2013) A survey of the natural variation in biomechanical and cell wall properties in inflorescence stems reveals new insights into the utility of Arabidopsis as a wood model. Funct Plant Biol 40: 662-676

Nakata MT, Takahara M, Sakamoto S, Yoshida K, Mitsuda N (2018) High-throughput analysis of Arabidopsis stem vibrations to identify mutants with altered mechanical properties. Front Plant Sci 9: 780

Niklas KJ, Spatz H (2012) Plant Physics. University of Chicago Press, Chicago

Nyquist H (1928) Certain topics in telegraph transmission theory. Am Instit Electric Eng Trans 47: 617-644

Onoda Y, Schieving F, Anten NP (2015) A novel method of measuring leaf epidermis and mesophyll stiffness shows the ubiquitous nature of the sandwich structure of leaf laminas in broad-leaved angiosperm species. J Exp Bot 66: 2487-2499

Paul-Victor C, Rowe N (2011) Effect of mechanical perturbation on the biomechanics, primary growth and secondary tissue development of inflorescence stems of Arabidopsis thaliana. Ann Bot 107: 209-218

Shah DU, Reynolds TPS, Ramage MH (2017) The strength of plants: Theory and experimental methods to measure the mechanical properties of stems. J Exp Bot 68: 4497-4516

Shannon CE (1949) Communication in the presence of noise. Proc IRE 37: 10-21

Spatz HC, Speck O (2002) Oscillation frequencies of tapered plant stems. Am J Bot 89: 1-11

Strabala TJ, Macmillan CP (2013) The Arabidopsis wood modelthe case for the inflorescence stem. Plant Sci 210: 193-205

The 1001 Genomes Consortium (2016) 1,135 Genomes Reveal the Global Pattern of Polymorphism in Arabidopsis thaliana. Cell 166: 481-491

Turner SR, Somerville CR (1997) Collapsed xylem phenotype of Arabidopsis identifies mutants deficient in cellulose deposition in the secondary cell wall. Plant Cell 9: 689-701

Zebrowski J (1991) The use of free vibrations to measure peduncle stiffness in triticale. J Exp Bot 42: 1207-1212

Zhu C, Ganguly A, Baskin TI, McClosky DD, Anderson CT, Foster C, Meunier KA, Okamoto R, Berg H, Dixit R (2015) The fragile fiber1 kinesin contributes to cortical microtubule-mediated trafficking of cell wall components. Plant Physiol 167: 780-792 